\title{
VALIDACIÓN DE LA WARWICK-ED!INBURGH MENTAL WELL-BEING SCALE (V5) EN UNA POBLACIÓN ESTUDIANTIL MEXICANA
}

\author{
Arturo Barraza Macias \\ Universidad Pedagógica de Durango \\ tbarraza-2017@hoitmail.com
}

\begin{abstract}
Recepción Artículo: 18 octubre 2020
Admisión Evaluación: 21 octubre 2020 Informe Evaluador 1: 25 noviembre 2020

Informe Evaluador 2: 23 noviembre 2020 Aprobación Publicación: 27 noviembre 2020
\end{abstract}

\section{RESUMEN}

En la presente investigación se buscó determinar el nivel de confiabilidad y las evidencias de validez basadas en la estructura interna, que respaldan el uso la Warwick-Edinburgh Mental Well-being Scale. Para el logro de este objetivo se llevó a cabo un estudio instrumental mediante la aplicación de la Warwick-Edinburgh Mental Well-being Scale a 760 alumnos de educación media superior de la ciudad de Durango, en Durango, México. Los resultados condujeron a una nueva versión de la escala con cinco ítems, diferente a Short Warwick Edinburgh Mental Well-Being Scale de siete ítems; esta nueva versión (WEMWBS-V5) presenta el mejor ajuste de las diferentes soluciones indagadas (2: 11.18; $p=.000$; RMSEA: 0.040). El Análisis Factorial Exploratorio posterior reporta un solo factor que explica el $47.18 \%$ de la varianza total. Su confiabilidad es de .81 en alfa de Cronbach y una confiabilidad por mitades, según la fórmula de Spearman-Brown de .81 para longitudes iguales.

Palabras clave: bienestar del estudiante; salud mental; psicometría

\section{ABSTRACT}

Validation of the Warwick-Edinburgh Mental Well-Being Scale (V5) in a Mexican student population. In the present research we sought to determine the level of reliability and evidence of validity, based on the internal structure, which support the use of the Warwick-Edinburgh Mental Well-being Scale. To achieve this goal, an instrumental study was carried out by applying the Warwick-Edinburgh Mental Well-being Scale to 760 upper secondary education students in the city of Durango, Durango, Mexico. The results led to a new version of the scale with five items, different from Seven Warwick Edinburgh Mental Well-Being Scale; this new version (WEMWBS-V5) presents the best adjustment of the different solutions investigated ( $2: 11.18, p=.000$, RMSEA: 0.040). The subsequent Exploratory Factor Analysis reports a single factor that explains $47.18 \%$ of the total 


\section{VALIDACIÓN DE LA WARWICK-EDINBURGH MENTAL WELL-BEING SCALE (V5) EN UNA}

POBLACIÓN ESTUDIANTIL MEXICANA

variance. Its reliability is .81 in Cronbach's alpha and a reliability by halves, according to the Spearman-Brown formula of .81 for equal lengths.

Keywords: student welfare; mental health; psychometry

\section{INTRODUCCIÓN}

El estudio sobre la enfermedad mental, orientado centralmente a su comprensión y tratamiento, ha tenido un fuerte desarrollo teórico y terapéutico desde la segunda mitad del siglo XX; esto, sin lugar a dudas, ha impactado, el desarrollo propio del campo de la psicología de la salud. Sin embargo, esta excesiva focalización en la enfermedad mental ha generado en el desarrollo teórico de la psicología de la salud dos grandes omisiones: buscar mejorar la vida de las personas y fomentar el genio (Seligman, 2002).

A partir del reconocimiento de estas omisiones surge el movimiento orientado a la psicología positiva que, como postulado central, afirma:

El interés ya no tiene por qué centrarse sólo en los problemas, las debilidades, los traumas, las inferioridades. Lo que se preconiza es que ha llegado el momento de abordar, con el mismo rigor científico de siempre y utilizando la misma metodología, las emociones placenteras, el crecimiento, la capacidad de hacer frente, de superación y la búsqueda de la felicidad, las virtudes, las potencialidades humanas, las fortalezas, Ios rasgos positivos del carácter etc. (Guillén, Botella \& Baños, 2016, p. 20).

Bajo este postulado, y en el marco de la denominada psicología positiva, se ha abordado el estudio de variables como el bienestar psicológico (Ryff, 1989), el bienestar subjetivo (Diener, Emmons, Larsen \& Griffin, 1985), las emociones positivas (Fredickson, 1998), y las fortalezas del carácter (Peterson \& Seligman, 2004), entre otras.

En el estudio de la variable bienestar, que es la de interés para la presente investigación, se pueden distinguir dos grandes tradiciones conceptuales: en la primera de ellas el individuo efectúa una valoración global de las diversas situaciones en las que se encuentra inmerso, tanto las que se consideran placenteras como las que no (tradición hedónica); esta tradición se identifica normalmente con el término bienestar subjetivo. En la segunda, intitulada tradición eudaimónica, se aborda el estudio del bienestar psicológico como un indicador empírico del funcionamiento positivo de un individuo, lo cual posibilita el óptimo desarrollo de las capacidades personales y por tanto, el crecimiento personal.

Estas tradiciones han dado lugar a múltiples investigaciones que abordan el bienestar subjetivo 0 el bienestar psicológico. Sin embargo, en el año 2007 un grupo de investigadores de las Universidades de Warwick y Edimburgo, con financiamiento del NHS Health Scotland diseñaron Warwick-Edinburgh Mental Well-being Scale (WEMWBS) para permitir la medición del bienestar mental en adultos del Reino Unido (Tennant et al. 2007), entendido este constructo como "a positive and sustainable mental state that allows individuals, groups and nations to thrive and flourish (Huppert, Baylis \& Keverne, 2004, p. 1).

Esta escala está centrada en los aspectos positivos de la salud mental, que incluye un aspecto hedónico (centrado en la experiencia subjetiva de felicidad y satisfacción con la vida) y un aspecto eudaimónico (centrado en el funcionamiento psicológico y la realización con uno mismo). En otras palabras, esta escala, y su constructo asociado, abordan las dos tradiciones conceptuales del bienestar ya mencionadas por lo que se convierte en una línea de investigación alternativa y de carácter más comprehensivo para el estudio del bienestar.

Desde su aparición en el año 2007 esta escala ha sido utilizada en múltiples estudios, demostrando con ellos su heurística positiva; lo mismo se estudia con relación a programa de mindfulness (Kuyken et al. 2013), los entornos residenciales psicosociales (Bond et al. 2012), el estilo de vida (Johnson et al. 2017), las fortalezas de carácter (Bauman \& Eiroa-Orosa, 2017), o las prácticas creativas (Saavedra et al. 2017), por mencionar solamente algunas variables. Sin embargo, es menester reconocer que una parte importante de estos estudios se ha focalizado en determinar sus propiedades psicométricas.

Inicialmente WEMWBS fue validada para su uso en el Reino Unido, para personas mayores de 16 años, pero en la actualidad se ha validado en diferentes poblaciones, como sería el caso de pacientes ingresados en las uni- 
dades de hospitalización, y los asistentes a las unidades de hospitalización y servicios ambulatorios (Ssw, Awy, Tks, Fsm, Aty, Rwt \& Dky, 2014), población psiquiátrica (Trousselard, et al. 2016), y estudiantes (Lloyd \& Devine, 2012).

Por su parte, en el año 2009, Stewart-Brown, et al. después de un proceso de validación con 779 participantes, decidieron eliminar siete ítems y dejar una escala de solo siete elemento que siguiera cubriendo el criterio unidimensional, surgiendo así la Short Warwick Edinburgh Mental Well-Being Scale (SWEMWBS) que ha sido también validada en posteriores estudios (Ssw et al. 2014).

De los diferentes procesos de validación realizados cobra especial interés para el presente trabajo el desarrollado con poblaciones de diferentes países; en lo particular esta escala se ha validado en países como Escocia (Stewart-Brown, et al. 2009), Irlanda del Norte (Lloyd \& Devine,2012), y Francia (Trousselard et al. 2016); en el caso latinoamericano solamente ha sido validada en Chile (Carvajal, Aboaja \& Alvarado, 2015), Brasil (Santos, Costa, Guilherme, Silva, Abentroth, Krebs \& Sotoriva, 2015) y Argentina (Serrani, 2015), por lo que cobra especial relevancia realizar su estudio de validación con población mexicana, siendo el objetivo de la presente investigación determinar el nivel de confiabilidad y las evidencias de validez que respaldan el uso la WarwickEdinburgh Mental Well-being Scale.

\section{MÉTODO}

\section{Participantes}

Para el logro del objetivo planteado se aplicó la Warwick-Edinburgh Mental Well-being Scale a una población de 760 alumnos de educación media superior de la ciudad de Durango, en Durango, México. La muestra invitada, un día previo a la aplicación, fue de 1200 alumnos, pero el día de la aplicación solo 760 alumnos presentaron el consentimiento informado debidamente firmado por su padre o tutor legal.

La selección de los participantes fue no probabilística, de tipo intencional y determinada por la accesibilidad a los mismos. Como la aplicación del cuestionario se realizó en las propias aulas escolares de los alumnos, y en días de clases, los criterios de inclusión fueron solamente el ser alumno de esa institución, haber asistido ese día a clases y presentar el consentimiento informado debidamente firmado por su respectivo padre o tutor legal, mientras que los criterios de exclusión fueron el no haber asistido ese día a clases o no presentar el consentimiento informado.

Con el objetivo de asegurar la protección de los participantes se realizaron cuatro acciones: a) de manera previa se les informó a los alumnos de la aplicación del cuestionario y se les entregó una hoja de consentimiento informado que se pidió enseñarán a sus padres y, en caso de estar de acuerdo en que su hijo respondiera el cuestionario, la firmaran; este consentimiento fue recogido al mismo tiempo que se entregaba el cuestionario y los alumnos que no lo llevaron no fueron objeto de la aplicación del instrumento, b) en el cuestionario no se les pidió el nombre y en la presentación del mismo se les aseguró la confidencialidad de los resultados; c) en el background solamente se solicitaron datos referentes a las variables sociodemográficas y/o situacionales que eran de interés para caracterizar la muestra y en ningún momento se les solicitó otro tipo de información que pudiera comprometer su anonimato; y d) en la presentación del cuestionario se les indicaba que su llenado era voluntario y estaban en libertad total de responderlo, o de no hacerlo. Con estas acciones se aseguró la confidencialidad de los resultados y se obtuvo el consentimiento informado. Las características sociodemográficas de la población encuestada son las siguientes: el 59.2\% pertenecen al sexo masculino y el $40.8 \%$ al sexo femenino; la edad mínima es de 15 años y la máxima de 21, siendo el promedio 16 años; el 72.4\% son alumnos del Centro de Bachillerato Tecnológico Industrial y de Servicios No. 89 y el 27.6\% el Centro de Bachillerato Tecnológico Industrial y de Servicios No. 110. 


\section{Materiales}

En la recolección de la información se utilizó Warwick-Edinburgh Mental Well-being Scale. Esta escala fue desarrollada a partir de: a) un panel de expertos de las áreas de psiquiatría, psicología, salud pública, ciencias sociales y promoción de la salud, todos ellos con experiencia en salud mental y bienestar, b) resultados de grupos focales y c) el análisis de las propiedades psicométricas de una escala ya existente denominada Affectometer (Tennant et al. 2007).

Existen dos traducciones al español de esta escala, pero en esencia solo presentan pequeñas variantes: a) una traducción es la utilizada por Carvajal et al. (2015) en población chilena y b) la otra traducción es la aplicada a población argentina por Serrani (2015). En la presente investigación se utilizó la aplicada a población chilena (Carvajal et al. 2015).

Esta escala consta de 14 ítems, todos ellos redactados como afirmaciones referentes a sentimientos o pensamientos que la persona ha tenido en las últimas dos semanas. Cada ítem tiene 5 opciones de respuesta, con una puntuación de 1 a 5 . El 1 indica que esa experiencia no le ocurre nunca y el 5 que le ocurre siempre. Todos los ítems están redactados con la misma direccionalidad, de forma tal que un mayor puntaje indica un mayor bienestar mental. A esta escala se le agregaron tres ítems en el background (sexo, edad e institución donde estudia).

En la aplicación de esta escala a la población chilena, que es el referente directo de la presente investigación, se reportaron los siguientes resultados:

- Un buen nivel de consistencia interna, con un de 0,875 para toda la escala.

- Una correlación positiva y estadísticamente significativa de test - retest $(r=0,556, p<0,001)$.

- Una solución con 3 factores, que explican el 56,8\% de la varianza, aunque finalmente recomiendan usarla de manera unifactorial.

\section{Procedimiento}

La presente investigación es de tipo instrumental y busca determinar la confiabilidad y las evidencias de validez de la Warwick-Edinburgh Mental Well-being Scale; tanto la confiabilidad como las evidencias de validez obtenidas son propiedades psicométricas del proceso de medición realizado por el instrumento objeto de validación.

Para el logro de este objetivo se desarrolló el siguiente procedimiento: Ios cuestionarios fueron administrados de manera individual en las instituciones donde estudian los encuestados; para esto se contó con la autorización de cada una de las autoridades correspondientes, después de que se les explicó las características de la investigación a realizar y asegurarles la no existencia de riesgos para los participantes; su aplicación tuvo una duración de aproximadamente 20 minutos en cada uno de los grupos donde se aplicó. En su aplicación se contó con el apoyo de colaboradores claves que fueron debidamente instruidos sobre las características de la investigación para que pudieran responder a cualquier duda que se generara al respecto.

\section{Análisis de datos}

Los alumnos encuestados la respondieron en su formato de papel. Una vez aplicada se construyó la base de datos en el programa SPSS versión 22. En esta base no hubo necesidad de imputar o substituir los datos perdidos ya que en ningún caso sobrepasó el 5\% (Littlewood \& Bernal, 2014). El ítem con menor cantidad de datos perdidos fue el número 8 con un .65\%, mientras que el ítem con mayor cantidad de datos perdidos fue el número 3 con un 2.1\%. Para el Análisis Factorial Confirmatorio se utilizó el programa LISREL 8.80 (Student).

El análisis de resultados se realizó en cuatro fases: a) en un primera fase se ejecutó el Análisis Factorial Exploratorio (método de máxima verosimilitud con rotación oblimin directo) que reportó tres factores; b) a partir de este resultado se efectuó el Análisis Factorial Confirmatorio; después de trabajar con diferentes modelos se optó por una solución de cinco ítems que mostraba un ajuste adecuado, c) en una tercera fase se hizo un análisis descriptivo de los diferentes ítems que conforman la versión de 5 ítems de la escala, y d) en una cuarta fase se realizó el análisis complementario de las propiedades psicométricas de la versión de 5 ítems de la escala a través 
de tres procedimientos: 1) obtención de la confiabilidad a través del alfa de Cronbach y de la confiabilidad por mitades, 2) Análisis de Consistencia Interna por medio del coeficiente $r$ de Pearson ( $p<.05)$, y 3) Análisis Factorial Exploratorio a partir del método de máxima verosimilitud con rotación oblimin directo.

\section{RESULTADOS}

\section{Análisis Factorial Exploratorio Inicial}

En el Análisis Factorial Exploratorio inicial la prueba de esfericidad de Bartlett fue significativa $(p<.001)$, y el test KMO de adecuación de la muestra alcanzó un valor .917. En la tabla de comunalidades se encontró que los ítems uno, cuatro y trece mostraban valores inferiores .3 (Tabla 1); el programa reportó la presencia de tres factores que explicaban el $44.64 \%$ de la varianza total (Tabla 2).

Tabla 1. Comunalidades de los ítems que conforman la WEMWBS

\begin{tabular}{|c|c|c|}
\hline Ítems & Inicial & $\underset{n}{\text { Extracció }}$ \\
\hline Uno & .280 & .262 \\
\hline Dos & .317 & .322 \\
\hline Tres & .344 & .407 \\
\hline Cuatro & .189 & .201 \\
\hline Cinco & .365 & .581 \\
\hline Seis & .406 & .520 \\
\hline Siete & .446 & .599 \\
\hline Ocho & .547 & .571 \\
\hline Nueve & .422 & .474 \\
\hline Diez & .527 & .610 \\
\hline Once & .380 & .437 \\
\hline Doce & .488 & .565 \\
\hline Trece & .227 & .224 \\
\hline Catorce & .469 & .477 \\
\hline
\end{tabular}

Tabla 2. Matriz Factorial de los ítems que conforman la WEMWBS

\begin{tabular}{lccc} 
& \multicolumn{3}{c}{ Factor } \\
\cline { 2 - 4 } \multicolumn{1}{c}{ Ítems } & $\mathbf{1}$ & $\mathbf{2}$ & $\mathbf{3}$ \\
\hline Uno & .483 & & \\
Dos & .538 & & \\
Tres & & .606 & \\
Cuatro & & .404 & \\
Cinco & & .756 & \\
Seis & & & -.716 \\
Siete & & & -.758 \\
Ocho & .707 & & \\
Nueve & .681 & & \\
Diez & .774 & & -.595 \\
Once & & & \\
Doce & .748 & & \\
Trece & .422 & & \\
Catorce & .685 & & \\
\hline
\end{tabular}




\section{Análisis Factorial Confirmatorio}

A partir de la identificación de estos tres factores se inició el trabajo con el Análisis Factorial Confirmatorio. Primeramente, se realizó el análisis con los tres factores; posteriormente con dos de ellos (factor uno y dos o factor uno y tres); luego se efectuó solo con el factor uno (que es el más numeroso y el que explica un mayor porcentaje de la varianza); y finalmente se exploraron varias soluciones eliminando algunos ítems que mejoraban el ajuste obtenido utilizando para ello los índices de modificación reportados por el propio programa.

La solución final de 5 ítems (2, 9, 10, 12 y 14) fue la que mostró el mejor ajuste (Tabla 3). Un buen ajuste se considera si el RMSEA < .05, (Bentler, 1990; Hu \& Bentler, 1999).

Tabla 3. Valores obtenidos en el Análisis Factorial Confirmatorio en las diferentes soluciones indagadas

\begin{tabular}{|c|c|c|c|c|c|c|}
\hline $\begin{array}{l}\text { Versión de la } \\
\text { escala }\end{array}$ & $\chi^{2}$ & df & P-value & RMSEA & $\begin{array}{c}\text { Confidence } \\
\text { Interval for } \\
\text { RMSEA } \\
\end{array}$ & $\begin{array}{c}\text { P-value for } \\
\text { test of close } \\
\text { Fit } \\
\end{array}$ \\
\hline Tres factores & 347.85 & 74 & 0.00000 & 0.070 & $0.063 ; 0.078$ & $\begin{array}{l}\text { (RMSEA } \\
<0.05 \text { ) } \\
=0.00\end{array}$ \\
\hline $\begin{array}{c}\text { Dos factores (1- } \\
2)\end{array}$ & 246.16 & 43 & 0.00000 & 0.070 & $0.069 ; 0.088$ & $\begin{array}{l}\text { (RMSEA } \\
<0.05 \text { ) } \\
=0.00\end{array}$ \\
\hline $\begin{array}{c}\text { Dos factores (1- } \\
3)\end{array}$ & 238.71 & 43 & 0.00000 & 0.078 & $0.068 ; 0.087$ & $\begin{array}{l}\text { (RMSEA } \\
<0.05 \text { ) } \\
=0.00\end{array}$ \\
\hline $\begin{array}{l}\text { Un factor }(8 \\
\text { ítems) }\end{array}$ & 150.14 & 20 & 0.00000 & 0.093 & $0.079 ; 0.11$ & $\begin{array}{l}\text { (RMSEA } \\
<0.05 \text { ) } \\
=0.00\end{array}$ \\
\hline $\begin{array}{l}\text { Un factor ( } 5 \\
\text { ítems) }\end{array}$ & 11.18 & 5 & 0.04801 & 0.040 & $0.0035 ; 0.073$ & $\begin{array}{l}\text { (RMSEA } \\
<0.05) \\
=0.64\end{array}$ \\
\hline
\end{tabular}

Figura 1. Modelo del Análisis Factorial Confirmatorio con 5 ítems

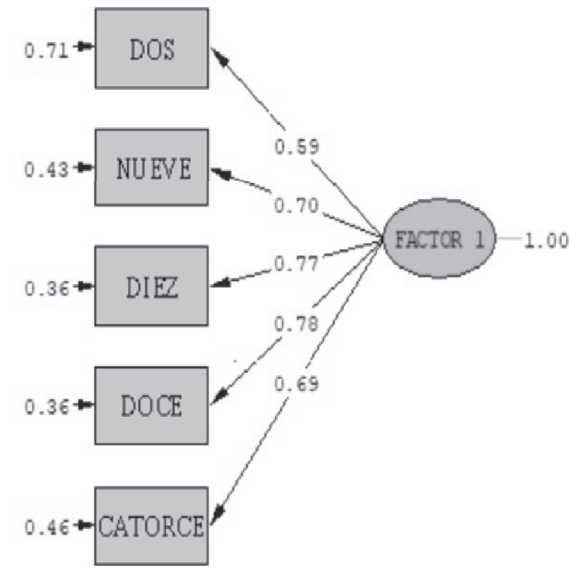

Chi-Square $=11.18, \mathrm{df}=5, \mathrm{P}$-value $=0.04801, \mathrm{RMSEA}=0.040$ 


\section{Análisis descriptivo}

Los datos descriptivos, obtenidos de esta versión de la escala, denominada WEMWBS-V5, se presentan en la tabla 4. El rasgo con la media más alta es el ítem 14: "He estado interesado en cosas nuevas", mientras que el que presenta la media más baja es el ítem 9: "Me he sentido cercano a las demás personas". La media general, en una escala de valores del 1 al 5 , es de 3.87 .

Tabla 4. Datos descriptivos de la WEMWBS-V5

\begin{tabular}{lcc}
\hline \multicolumn{1}{c}{ ítems } & x & s2 \\
\hline Dos & 3.81 & .982 \\
Nueve & 3.58 & .918 \\
Diez & 3.85 & .928 \\
Doce & 3.99 & .930 \\
Catorce & 4.14 & .906 \\
\hline
\end{tabular}

En este análisis se siguió el procedimiento establecido por Salkind (1999) obteniendo como resultado que todos los ítems se correlacionan de manera positiva con la media general de cada encuestado, siendo el valor $r$ menor .673 y el mayor de .797.

\section{Confiabilidad}

La WEMWBS-V5 presenta una confiabilidad de .81 en alfa de Cronbach (con un 95\% de intervalo de confianza en el coeficiente de correlación intraclase para un modelo de efectos aleatorio de un factor: $p<.01$ ) y una confiabilidad por mitades, según la fórmula de Spearman-Brown de .81 para una longitud desigual (con un 95\% de intervalo de confianza en el coeficiente de correlación intraclase para un modelo de efectos aleatorio de un factor: $p<.01)$.

Como complemento a estos niveles de confiabilidad se obtuvo el nivel de confiablidad en alfa de Cronbach en caso de eliminar un ítem y como resultado se pudo establecer que en el caso del ítem dos el nivel de confiabilidad se mantiene en .81, mientras que en el resto de los ítems baja a .77, .76 ó .75; en ninguno de los casos se incrementa el nivel de confiabilidad.

\section{Análisis Factorial Exploratorio Final}

En este Análisis Factorial Exploratorio la prueba de esfericidad de Bartlett fue significativa $(p<.001)$, y el test KMO de adecuación de la muestra alcanzó un valor .837. En la tabla de comunalidades se encontró que solamente el ítem dos mostraba un valor inferior .3; el programa reportó la presencia de un solo factor que explicaba el $47.18 \%$ de la varianza total. Las cargas factoriales de cada ítem se presentan en la tabla 5.

Tabla 5. Matriz Factorial de los ítems que conforman la WEMWBS-V5

\begin{tabular}{lc}
\hline & Factor \\
\cline { 2 - 2 } \multicolumn{1}{c}{ ítems } & $\mathbf{1}$ \\
\hline Dos & .514 \\
Nueve & .697 \\
Diez & .765 \\
Doce & .762 \\
Catorce & .665 \\
\hline
\end{tabular}




\section{Comentarios}

En la actualidad existe un marcado interés por el estudio, y la promoción, de un estado de bienestar en las personas, entendido éste como aquel "en el que el individuo alcanza su potencial, puede lidiar con el estrés habitual de la vida, y es capaz de proporcionar su contribución a su comunidad" (Organización Mundial de la Salud, 2016, párr.. 2).

Este interés motivó el diseño y validación de la Warwick-Edinburgh Mental Well-being Scale (Tennant et al. 2007), la cual ha mostrado su potencial heurístico al medir y relacionar su constructo subyacente con múltiples variables. La mayor parte de estos estudios se han realizado en los países europeos y en Norteamérica, descuidándose su estudio y validación en los países latinoamericanos, salvo tres excepciones: Chile, Argentina y Brasil.

Con la intención de avanzar en su estudio, en población mexicana, se planteó su validación en una población estudiantil de una ciudad del norte de México, sin embargo los resultados condujeron a una nueva versión de la escala con cinco ítems, diferente a Short Warwick Edinburgh Mental Well-Being Scale de siete ítems.

Esta nueva versión (WEMWBS-V5) presenta el mejor ajuste de las diferentes soluciones indagadas (2: 11.18; $p=.000$; RMSEA: 0.040); así mismo, el Análisis Factorial Exploratorio reporta un solo factor que explica el $47.18 \%$ de la varianza total.

El Análisis de Consistencia Interna mostró que todos los ítems tienen una misma direccionalidad, ya que es una medida que se concentra enteramente en los aspectos positivos de la salud mental. Su confiabilidad es de .81 en alfa de Cronbach y una confiabilidad por mitades, según la fórmula de Spearman-Brown de .81 para Iongitudes iguales.

La aplicación de la Warwick-Edinburgh Mental Well-being Scale a la población latinoamericana viene precedida de su respectivo proceso de validación (Tennant et al. 2007) y de su traducción y adaptacion a diferentes idiomas; en el caso latinoamericano, y específicamente en los países de habla hispana, se ha validado solamente en Chile (Carvajal et al. 2015) y Argentina (Serrani, 2015).

En el primer caso se realiza un Análisis Factorial Exploratorio, mientras que en el segundo se realiza el Análisis Factorial Exploratorio y Confirmatorio; en la presente investigación se realizan ambos tipos de análisis factoriales y, a diferencia de los estudios precedentes, se llega a presentar una propuesta de versión breve de la escala de solo cinco items.

Esta nueva propuesta de version breve de la Warwick-Edinburgh Mental Well-being Scale abre otra línea de investigación para posteriores estudios. Tal vez estaríamos hablando de una version breve para población de habla hispana.

La adecuada medición de la variable Bienestar Mental, en lo general, adquiere relevancia en dos niveles discursivos: el explicativo y el prescriptivo. A nivel explicativo permitirá la realización de más investigaciones que posibiliten acumular mayor evidencia empírica y, por ende, contribuya al desarrollo y contrastación de modelos teóricos. A nivel prescriptivo, el conocimiento generado permitirá el desarrollo de medidas preventivas, vía la formulación de políticas publicas, para la población.

Este avance, en ambos niveles discursivos, depende de la existencia de instrumentos de medición que generen medidas confiables y válidas de la variable Bienestar Mental y, en ese sentido, la realización de este estudio coadyuva a tal fin.

La principal limitación del presente estudio se deriva de la muestra circunscrita a adolescentes escolarizados de educación media superior de carácter técnico, por lo que se recomienda continuar su proceso de validación en poblaciones diferentes; así mismo, se recomienda realizar procesos de validación de la WEMWBS-V5.

\section{REFERENCIAS BIBLIOGRÁFICAS}

Baumann, D. \& Eiroa-Orosa, F.J. (2017). Mental Well-Being in Later Life: The Role of Strengths Use, Meaning in Life, and Self-Perceptions of Ageing. International Journal of Applied Positive Psychology, 1-19. doi: 10.1007/s41042-017-0004-0. 
Bentler, P. M. (1990). Comparative fit indexes in structural models. Psychological Bulletin, 107(2), 238-246. doi:10.1037/0033-2909.107.2.238 238-246.

Bond, L., Kearns, A., Mason, P., Tannahill, C., Egan, M. \& Whitely, E. (2012). Exploring the relationships between housing, neighbourhoods and mental wellbeing for residents of deprived areas. BMC Public Health, 12(48), 1-14. doi: 10.1186/1471-2458-12-48.

Carvajal, D., Aboaja, A. y Alvarado, R. (2015). Validación de la Escala De Bienestar Mental de WarwickEdinburgo, en Chile. Revista de Salud Pública, (XIX)1, 13-21. Recovered from http://www.saludpublica.fcm.unc.edu.ar/sites/default/files/RSP15_1_04_\%20art1.pdf

Diener, E., Emmons, R., Larsen, R.J. \& Griffin, S. (1985). The Satisfaction with Life Scale. Journal of Personality Assessment, 49, 71-75. doi: 10.1207/s15327752jpa4901_13.

Fredrickson, B.L. (1998). What good are positive emotions? Review of General Psychology, 2(3), 300-319. doi: 10.1037/1089-2680.2.3.300.

Guillén, V., Botella, C. y Baños, R. (2016). Psicología clínica positiva y tecnologías positivas. Papeles del Psicólogo, 38(1),19-25. doi: https://doi.org/10.23923/pap.psicol2017.2817.

Hu, L. \& Bentler, P.M. (1999). Cutoff criteria for fit indexes in covariance structure analysis: Conventional criteria versus new alternatives. Structural Equations Modeling, A Multidisciplinary Journal, 6(1), 1-55. doi: 10.1080/10705519909540118.

Huppert, F.A., Baylis, N. \& Keverne, B. (2004). Why do we need a science of wellbeing? Philosophical Transactions of the Royal Society of London, 359, 1331-1332. doi:10.1098/rstb.2004.1519.

Johnson, R., Robertson, W., Towey, M., Stewart-Brown, S. \& Clarke, A. (2017). Changes over time in mental wellbeing, fruit and vegetable consumption and physical activity in a community-based lifestyle intervention: a before and after study. Public Health, 146, 118 -125. doi:10.1016/j.puhe.2017.01.012.

Kuyken, W., Weare, K., Ukoumunne, O.C., Vicary, R., Motton, N., Burnett, R. ... Huppert, F. (2013). Effectiveness of the mindfulness in schools programme: non-randomised controlled feasibility study. The British Journal of Psychiatry, 203, 126-131. doi: 10.1192/bjp.bp.113.126649.

Littlewood, H. F. \& Bernal, E.R. (2014). Mi primer modelamiento de ecuaciones estructurales LISREL. México: Cincel. Recuperado de https://www.researchgate.net/publication/316450535_Mi_primer_modelamiento_de_ecuaciones_estructurales_2a_edicion.

Lloyd, K. \& Devine, P. (2012). Psychometric properties of the Warwick-Edinburgh Mental Well-being Scale (WEMWBS) in Northern Ireland. Journal of Mental Health, 21(3), 257-263. doi: 10.3109/09638237.2012.670883.

Organización Mundial de la Salud. (2016). Salud mental: fortalecer nuestra respuesta. Nota descriptiva. Recuperado de https://www.who.int/es/news-room/fact-sheets/detail/mental-health-strengthening-our-response

Peterson, C., \& Seligman, M. E. P. (2004). Character strengths and virtues: A handbook and classification. New York: Oxford University Press.

Ryff, C. D. (1989). Happiness is everything, or is it? Explorations on the meaning of psychological wellbeing. Journal of Personality and Social Psychology, 57, 1069-1081. doi: 10.1037/0022-3514.57.6.1069.

Saavedra, J., Pérez, E., Crawford, P. \& Arias, S. (2017). Recovery and creative practices in people with severe mental illness: evaluating well-being and social inclusion. Disability And Rehabilitation, $0(0,0), 1-10$. doi: 10.1080/09638288.2017.1278797.

Salkind, N.J. (1999). Métodos de Investigación. México: Prentice Hall.

Santos, J.J., Costa, T.A., Guilherme, J.H., Silva, W.C., Abentroth, L.L.R., Krebs, J. \& Sotoriva, P. (2015). Adaptation and cross-cultural validation of the Brazilian version of the Warwick-Edinburgh mental well-being scale. Revista da Associação Médica Brasileira, 61(3), 209-214. doi: 10.1590/1806-9282.61.03.209. 
Seligman, M.E.P. (2002). Positive Psychology, positive prevention and positive therapy. En C.R. Snyder \& S.J. López (Eds.). Handbook of positive psychology (pp.3-9). New York, NY: Oxford University Press.

Serrani, D. (2015). Traducción, adaptación al español y validación de la Escala de Bienestar Mental de WarwickEdinburgh en una muestra de adultos mayores argentinos. Acta Colombiana de Psicología, 18(1), 79-93. doi: http://www.dx.doi.org/10.14718/ACP.2015.18.1.8.

Ssw, N., Awy, L., Tks, L., Fsm, C., Aty, W., Rwt, L. \& Dky, T. (2014). Translation and validation of the chinese version of the short Warwick-Edinburgh Mental Well-Being Scale for patients with mental illness in Hong Kong. East Asian Archives Psychiatry, 24(1), 3-9. Recovered from http://easap.asia/journal_file/1401_V24N1_p3.pdf.

Stewart-Brown, S., Tennant, A., Tennant, R., Platt, S., Parkinson, J. \& Weich, S. (2009). Internal construct validity of the Warwick-Edinburgh Mental Well-being Scale (WEMWBS): a Rasch analysis using data from the Scottish Health Education Population Survey. Health and Quality of Life Outcomes, 7(15), 1-8. doi: 10.1186/1477-7525-7-15.

Tennant, R., Hiller, L., Fishwick, R., Platt, S., Joseph, S., Weich, S., ... Stewart-Brown, S. (2007). The WarwickEdinburgh Mental Well-being Scale (WEMWBS): development and UK validation. Health and Quality of Life Outcomes, 5(63), 1-7. doi:10.1186/1477-7525-5-63.

Trousselard, M., Steiler, D., Dutheil, F., Claverie, D., Canini, F., Fenouillet, F., ... Franck, N. (2016). Validation of the Warwick-Edinburgh Mental Well-Being Scale (WEMWBS) in French psychiatric and general populations. Psychiatry Research, 245, 282-290. doi:10.1016/j.psychres.2016.08.050 